\title{
Milnacipran poorly modulates pain in patients suffering from fibromyalgia: a randomized double-blind controlled study
}

This article was published in the following Dove Press journal:

Drug Design, Development and Therapy

\author{
Gisèle Pickering ${ }^{1,2}$ \\ Nicolas Macian ${ }^{2}$ \\ Noémie Delage ${ }^{3}$ \\ Pascale Picard ${ }^{3}$ \\ Jean-Michel Cardot ${ }^{4}$ \\ Sophia Sickout-Arondo ${ }^{2}$ \\ Fatiha Giron ${ }^{2}$ \\ Christian Dualé2 \\ Bruno Pereira $^{5}$ \\ Fabienne Marcaillou ${ }^{3}$ \\ 'University Clermont Auvergne \\ Neurodol, Clermont-Ferrand, France; \\ ${ }^{2}$ Clinical Pharmacology Department \\ $\mathrm{CPC/CIC}$ Inserm 1405, University \\ Hospital, Clermont-Ferrand, France; \\ ${ }^{3}$ Pain Clinic, CHU Clermont- \\ Ferrand, Clermont-Ferrand, France; \\ ${ }^{4}$ University Clermont Auvergne \\ MEDIS, CHU Clermont-Ferrand, \\ Clermont-Ferrand, France; ${ }^{5} \mathrm{DRCl}$, \\ $\mathrm{CHU}$ Clermont-Ferrand, Clermont- \\ Ferrand, France
}

Correspondence: Gisèle Pickering Centre de Pharmacologie Clinique, Bâtiment 3C, ClC Inserm I405, CHU Clermont-Ferrand, BP 69, ClermontFerrand Cedex I F-63003, France

Tel +33473178416

Fax +33473178412

Email gisele.pickering@uca.fr
Introduction: Fibromyalgia is characterized by widespread and chronic pain, and its prevalence is increasing worldwide. Milnacipran, an antidepressant, is often prescribed for fibromyalgia with a possible beneficial effect on central pain modulation. The aim of this study was to evaluate if milnacipran could modify the status of conditioned pain modulation (CPM) in patients suffering from fibromyalgia.

Design and setting: Randomized, double-blind controlled trial.

Subjects and methods: Women with fibromyalgia received milnacipran $100 \mathrm{mg}$ or placebo. The primary end point was the evolution of CPM with treatments after a 30-second painful stimulus. Secondary outcomes included the predictability of milnacipran efficacy from CPM performance, evolution of global pain, mechanical sensitivity, thermal pain threshold, mechanical allodynia, cognitive function, and tolerance.

Results: Fifty-four women with fibromyalgia (46.7 \pm 10.6 years) were included and randomized, and 24 patients were analyzed in each group. At inclusion, CPM was dysfunctional $\left(\mathrm{CPM}_{30}=-0.5 \pm 1.9\right)$, and global pain was $6.5 \pm 1.8$. After treatment, there was a nonsignificant $\mathrm{CPM}$ difference between milnacipran and placebo $\left(\mathrm{CPM}_{30}=-0.46 \pm 1.22 \mathrm{vs}-0.69 \pm 1.43\right.$, respectively, $p=0.55)$ and $18.8 \%$ vs $6.3 \%(p=0.085)$ patients did reactivate CPM after milnacipran vs placebo. Initial CPM was not a predictor of milnacipran efficacy. Global pain, mechanical and thermal thresholds, allodynia, cognition, and tolerance were not significantly different between both groups.

Conclusion: Milnacipran did not display a significant analgesic effect after 1-month treatment, but the tendency of milnacipran to reactivate CPM in a number of patients must be explored with longer treatment duration in future studies and pleads for possible subtypes of fibromyalgia patients.

Keywords: fibromyalgia, CPM, milnacipran, antidepressants, pain

\section{Introduction}

Fibromyalgia (FM) is characterized by diffuse chronic pain affecting $0.5 \%-5 \%$ of the general population, with a female predominance. ${ }^{1-4}$ Symptoms include joint and muscle pain associated with fatigue, migraine, sleep disorder, depression, and/or irritable bowel syndrome. ${ }^{5-10}$ Symptom presentation varies greatly between individuals, with a wide variety of clinical, physical, social, and psychological repercussions and of therapeutic responses. Studies on FM pathophysiology report hyperalgesia to experimental stimuli, ${ }^{10,11-14}$ central nervous system abnormalities and cerebral changes, ${ }^{15-19}$ autonomic and neuroendocrine system abnormalities, ${ }^{20}$ genetic factors, ${ }^{21}$ and microbiota specificities. ${ }^{22}$ 
Alteration in the central modulation of pain and diffuse noxious inhibitory controls has been demonstrated in FM patients ${ }^{19,23-25}$ using an experimental psychophysics technique, conditioned pain modulation (CPM). ${ }^{26-29}$ Dysfunction of neurotransmitters has also been described, ${ }^{30-39}$ justifying the use of antidepressants in FM patients. Milnacipran inhibits the recapture of monoamines at presynaptic level, ${ }^{40}$ with an increase in postsynaptic concentrations with CPM activation and diminution of pain. ${ }^{41-43}$ Noradrenaline has a central analgesic action with a synergistic effect with serotonin, ${ }^{41,44,45}$ and this would make milnacipran a good candidate for pain treatment. Milnacipran is recommended to treat FM in several countries, including North America and Russia. ${ }^{46}$

Duloxetine and milnacipran (both serotonin and norepinephrine reuptake inhibitor antidepressants) have shown a $30 \%$ reduction in pain, but in only $40 \%$ of the patients. ${ }^{47-50}$

Considering that CPM is poorly functional in FM patients, ${ }^{51}$ the hypothesis is that milnacipran might reactivate the efficacy of pain modulation and restore the lost functionality of these central pathways. The present study aims to evaluate CPM efficacy with milnacipran compared to placebo after 1-month treatment.

\section{Subjects and methods Study design}

This study is a prospective, randomized, controlled doubleblind clinical trial with 2 parallel groups, milnacipran vs placebo in FM patients. It was carried out in the Pain Clinic/Clinical Pharmacology Center, University Hospital, Clermont-Ferrand, France, according to Good Clinical Practice. The Research Ethics Committee (CPP South East VI) gave its approval on October 18, 2012 (leading ethics committee number institutional review board [IRB] 00008526/ AU987); the national Drug agency gave its approval on September 25, 2012 (EudraCT number 2012-003222-24); and this trial is registered in ClinicalTrials.gov (trial number NCT01747044).

\section{Study population}

At inclusion, written informed consent was obtained from all patients. Eligibility criteria were verified with a complete clinical examination matching the 2010 American College of Rheumatology definition of FM. ${ }^{52}$ Patients were included if they fulfilled the following criteria: age $>18$ years with a confirmed diagnosis of FM, able to understand and follow the study protocol and have given informed consent. Patients were excluded if they met any of the following criteria: contraindication to milnacipran treatment, hypertension or heart disease, known renal impairment, patients with a medical and/or surgery history noncompatible with the study, and patients with a psychiatric disorder or with suicidal behavior/ significant suicide ideas. This study also excluded patients with diabetes; patients taking alcohol, hypnotics, analgesics, and opioids; patients taking diuretics or a treatment inducing hyponatremia, nonsteroidal anti-inflammatory drugs, oral anticoagulants, aspirin, or other drugs that are likely to induce bleeding, drugs with serotonin and noradrenalin reuptake inhibition, digitalis, CYP1A2 inhibitors, and 5HT1D agonist. Also excluded were women of childbearing potential not using an effective contraceptive, pregnant or breastfeeding, taking part in another interventional trial or unable to understand patient information and informed consent form.

\section{Study treatment}

Patients were selected, included, and then randomized to oral milnacipran (IXEL ${ }^{\circledR}$, batch number G00771, G00793, G00794) or oral placebo for 1-month treatment (Figure 1), with the following titration: $50 \mathrm{mg}$ on the first 3 days, $75 \mathrm{mg}$ from Day 4 to Day 6, and $100 \mathrm{mg}$ from Day 7 to 1 month (M1), and the last treatment administration was on the evening before M1 visit where evaluations and tests, similar to inclusion, were carried out. The capsules of placebo were made and packaged by the pharmacy department (authorization number 2015-704) of the University Hospital of Clermont-Ferrand, France.

To maintain a good compliance and to verify adverse events (AEs), patients were called once a week by a clinical research assistant. Treatment allocation followed a predefined randomization plan and was conducted by a person independent from the protocol. The randomization sequence was generated using random blocks.

\section{Objectives and study outcome variables}

The primary objective of this trial was to assess if milnacipran has an influence on pain modulation profile by comparing CPM changes after a 30 -second stimulus $\left(\mathrm{CPM}_{30}\right)$ in milnacipran and placebo groups. The secondary objectives were to 1) compare $\mathrm{CPM}$ changes after 10 seconds $\left(\mathrm{CPM}_{10}\right)$ in milnacipran and placebo groups, 2) assess the evolution of pain thresholds with milnacipran, 3) evaluate if CPM at inclusion could be predictive of milnacipran efficacy in FM, 4) explore the impact of milnacipran on cognitive parameters (comprehension tests, executive function, memory, attention, and decision making), and 5) evaluate the tolerance to milnacipran. 


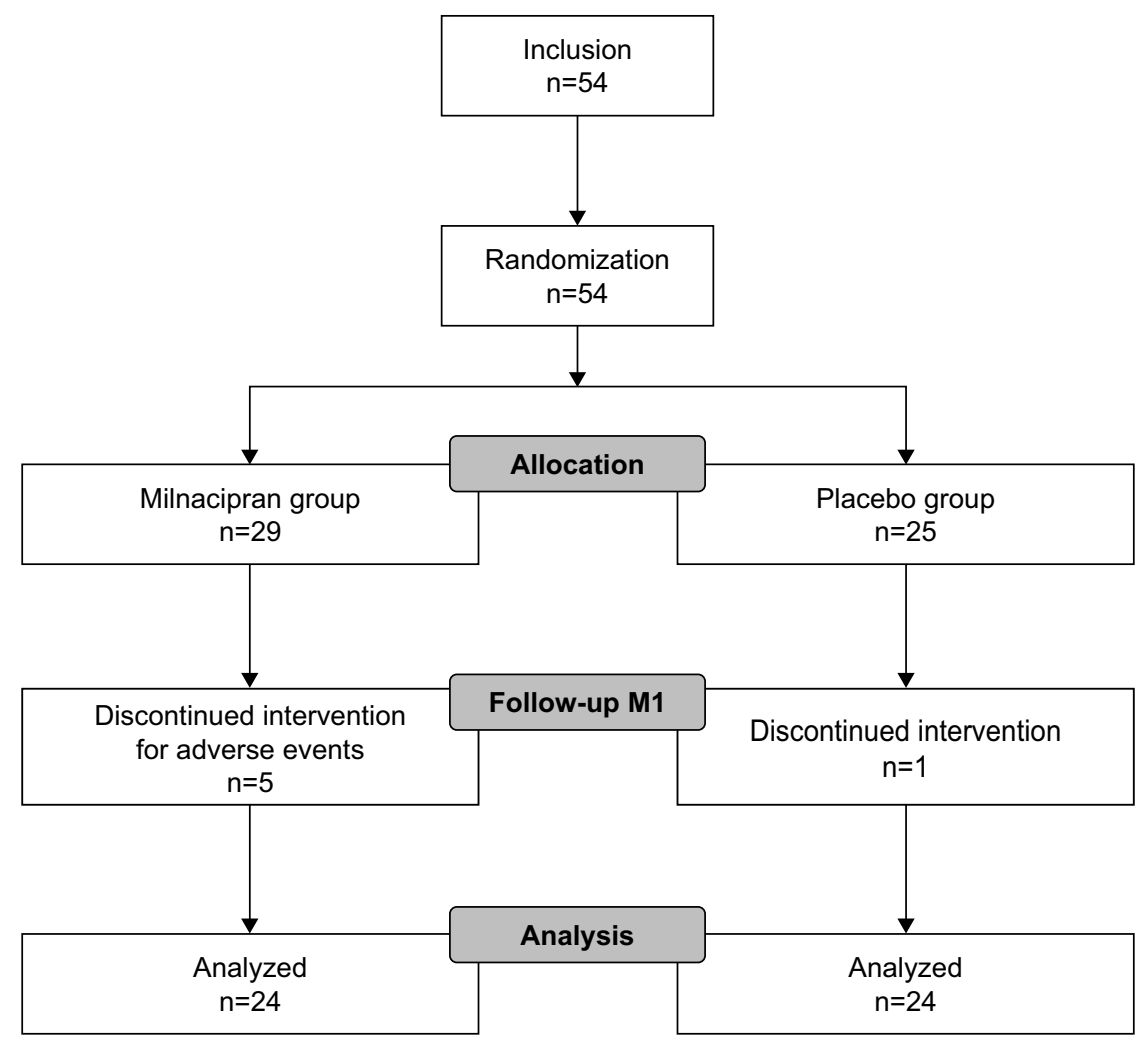

Figure I CONSORT study flow chart.

\section{Outcome measures}

\section{Pain evaluation and pain responders}

Pain intensity was measured by the Numeric Pain Rating Scale (NRS), and patients verbally rated the intensity of pain on a scale from "0" (no pain) to "10" (worst pain possible). "Pain responders" were patients presenting a 30\% improvement in pain score at M1.

\section{Assessment of pain thresholds to a thermal stimulus}

The advanced thermal stimulation (ATS) thermode $(30 \times 30$ $\mathrm{mm}$ ) (Medoc Ltd., Ramat Yishai, Israel) connected to Pathway-Medoc ${ }^{\circledR}$ was applied to the volar side of the patientdominant forearm. From the baseline value of $32^{\circ} \mathrm{C}$, the Pathway-Medoc delivers an adjustable temperature peak (in cold and heat, depending on a regular slope of $1^{\circ} \mathrm{C}$ ) and is controlled by rapid feedback. This device was used to evaluate the heat pain threshold (HPT) and cold pain threshold by calculating the mean of 3 measures. This technique has been used successfully in neurophysiology studies in humans ${ }^{53}$ and showed similar results than those obtained with a laser. ${ }^{54}$

\section{Test stimulus intensity "pain 60/stimulus"}

The ATS thermode was applied to the volar side of the patient-dominant forearm. From the baseline value of $32^{\circ} \mathrm{C}$, the Pathway-Medoc delivered a series of peaks starting from $1{ }^{\circ} \mathrm{C}$ above HPT. Then the temperature was increased by $1^{\circ} \mathrm{C}$ until the patient rated the pain intensity equivalent to $6 / 10$ on the NRS. The temperature (T6/10) corresponding to this $6 / 10$ rated pain intensity was used for the CPM test.

\section{Conditioned pain modulation}

The ATS thermode was applied to the volar side of the patientdominant forearm. The Pathway-Medoc delivered stimulation at the predetermined $\mathrm{T} 6 / 10$ for a duration of 10 seconds, and the patient rated her pain using the NRS. Then, the PathwayMedoc delivered a stimulus at the same temperature for a duration of 30 seconds, and the patient rated her pain with NRS. After these stimuli (15 minutes), the patient put the nondominant hand for 60 seconds in a water bath at $46.5^{\circ} \mathrm{C}$. After having dried her hand, the patient had another sequence of stimuli of 10 and 30 seconds with NRS pain evaluation. The aim of this test was to trigger the stimulation of inhibitory pain descending pathways. If these pathways were functional, the second series of stimuli would be less painful than the first series because of the inhibitory effect on pain as described in a number of chronic pain situations including FM..$^{53,55,56}$ CPM is calculated as the difference (for 10- and 30-second stimulation $\left[\mathrm{CPM}_{10}\right.$ and $\mathrm{CPM}_{30}$, respectively]) between the 
NRS pain scores rated after and before immersion of the nondominant hand in the water bath at $46.5^{\circ} \mathrm{C}$. Delta CPM is the CPM difference between M1 and inclusion.

\section{Pain modulation profile}

Laboratory-based pain modulation using the CPM test as described previously allows to explore a pain modulation profile $^{57}$ based on the CPM response $(<0,0$, or $>0)$ and to classify patients on a scale with an "inhibitory" status (pain inhibition, $\mathrm{CPM}<0$ ), a "facilitatory" status (pain facilitation, when patients have a $\mathrm{CPM}>0$ ), and a "neutral" status (no inhibition or facilitation, with $\mathrm{CPM}=0$ ).

\section{Cantab $^{\circledR}$ tests}

The Cambridge Neuropsychological Test Automated Battery (Cantab, Cambridge, UK $)^{58}$ is a battery of 22 neuropsychological tests administered to subjects using a touch screen computer and a press-pad. The selected tests are as follows: spatial memory was evaluated with the Stockings of Cambridge test, a test of spatial planning and working memory; choice reaction time was an attention test, which measures the speed of response and movement in 5-choice paradigm; decision making and risk-taking outside a learning context with Cambridge Gambling Task, this test dissociates risk-taking from impulsivity; semantic memory was explored with the Graded Naming Test that gives a measure of semantic memory by assessing object-naming ability.

\section{Mechanical allodynia}

Mechanical allodynia was assessed by using a standardized brush (Somedic, Hörby, Sweden) with moving innocuous stimuli applied in a balanced manner on the volar side of the patient nondominant forearm, and the patient rated pain on a " $0-10$ " NRS.

\section{Mechanical sensitivity}

Mechanical sensitivity was measured with a standardized set of modified von Frey ${ }^{\circledR}$ hair (Optihair2-Set; Marstock Nervtest, Schriesheim, Germany) that exert forces upon bending between 0.25 and $512 \mathrm{mN}$. von Frey hair was applied 3 times to the volar side of the patient nondominant forearm with increasing hair diameter until the patient felt 2 of the 3 stimuli. This hair diameter was considered as the mechanical sensitivity threshold.

\section{Temporal summation}

Temporal summation was realized using von Frey hair (180 g, size 6.45), applied to the volar side of the patient nondominant forearm. The 6.45 von Frey hair was applied once, and the patient rated her pain with NRS. The 6.45 von Frey hair was then applied 10 times at a rate of $1 \mathrm{~Hz}(1$ stimuli per second), and the patient rated her pain. Temporal summation was calculated as the difference (for 1 and 10 stimuli) between the NRS pain scores.

\section{Statistical analysis}

According to our previous works and literature, ${ }^{28}$ we estimated that a sample size of $n=24$ patients per randomized group would provide $90 \%$ statistical power to detect an absolute difference of 1.5 points (SD of CPM at 1.6) in the primary outcome. A 2 -sided $p$-value of $<0.05$ was considered to indicate statistical significance for the change in CPM effect between milnacipran and placebo. Statistical analysis was conducted on per protocol analysis.

Baseline characteristics have been presented for each randomized group as the mean $\pm \mathrm{SD}$ or the median (interquartile range) according to statistical distribution for continuous data and as the number of patients and associated percentages for categorical parameters. Comparisons between independent groups have been analyzed using the chi-squared or Fisher's exact test for categorical variables and Student's $t$-test or Mann-Whitney test for quantitative parameters (notably primary outcome, temporal summation, test stimulus intensity pain 60/stimulus, pain rating on the NRS, cognitive parameters), with normality verified by the Shapiro-Wilk test and homoscedasticity by the Fisher-Snedecor test. As suggested by Vickers and Altman, ${ }^{59}$ the primary analysis has been completed by analysis of covariance considering CPM at 1 month as a dependent variable ${ }^{28}$ and group and baseline value of CPM as independent parameters. No missing data occurred in this work. Analyses were conducted using SAS9.4 for Windows (SAS, Cary, NC, USA).

\section{Results}

\section{Patients and baseline characteristics}

Fifty-four women with FM (46.7 \pm 10.6 years old) were included between April 2013 and December 2016 and 48 (per protocol analysis) were analyzed (Figure 1). FM duration was $91.3 \pm 86.0$ months in the milnacipran group vs $52.4 \pm 33.3$ months in the placebo group ( $p=0.048$ ). Six patients (11\%) discontinued the study, 5 patients $(9 \%)$ in the milnacipran group for adverse events and 1 patient $(2 \%)$ in the placebo group for personal reasons. Characteristics of the population at inclusion are indicated in Table S1. At inclusion, CPM was dysfunctional $\left(\mathrm{CPM}_{10}=-0.1 \pm 1.6\right.$, $\left.\mathrm{CPM}_{30}=-0.5 \pm 1.9\right)$. 
Considering $\mathrm{CPM}_{30}$, at baseline, global distribution of pain modulation profile was of $40 \%$ inhibitor, $29 \%$ facilitator, and $31 \%$ neutral. In the milnacipran group, $12.5 \%$ were inhibitor, $18.8 \%$ facilitator, and $18.8 \%$ neutral. In the placebo group, $27.1 \%$ were inhibitor, $10.4 \%$ facilitator, and $12.5 \%$ neutral (percentage of total number of subjects $n=48$ ). No significant difference in the distribution was observed $(p=0.131)$.

\section{Efficacy results}

At M1, $\mathrm{CPM}_{30}$ was similar in milnacipran and placebo groups $(-0.46 \pm 1.22$ vs $-0.69 \pm 1.43$, respectively, $p=0.552$; Figure 2$)$. Similarly, no difference was found for $\mathrm{CPM}_{10}$ (respectively for milnacipran and placebo groups, $0.0 \pm 1.44 \mathrm{vs}-0.27 \pm 1.05$, $p=0.462$ ). The differences between inclusion and M1 were also similar in milnacipran and placebo groups, both for $\mathrm{CPM}_{10}(0.06 \pm 1.98 \mathrm{vs}-0.21 \pm 1.59$, respectively, $p=0.604)$ and $\mathrm{CPM}_{30}(-0.44 \pm 2.00$ vs $0.35 \pm 2.1$, respectively, $p=0.188)$.

Regarding the evolution of pain modulation profile with treatment, $18.8 \%$ patients turned to an "inhibitory" profile in the milnacipran group and $6.3 \%$ in the placebo group ( $p=0.085$; Figures 3 and 4 ).

Considering pain scores, no difference was observed between the milnacipran and placebo groups, either for raw values $(5.3 \pm 2.5$ vs $5.6 \pm 1.8$, respectively, $p=0.560)$ or for differences from baseline $(-1.0 \pm 2.1$ vs $-1.0 \pm 1.7$, respectively, $p=0.971$; Figure 5), whereas a significant reduction in pain scores was observed from baseline to M1 in both groups ( $p=0.029$ and $p=0.010$ ). Regarding patients considered as "pain responders", $29 \%$ of patient reported a $30 \%$ improvement of pain in the milnacipran group vs $25 \%$ in the placebo group ( $p=0.745)$.

Thermal thresholds (cold and hot), mechanical allodynia, mechanical sensitivity threshold, temporal summation, and cognitive tests were not significantly different between both groups at M1 (Tables S2 and S3).

FM patients had cognitive impairment when compared with healthy volunteers of another study in our center (same age and sex) (Corriger et al, unpublished data, 2017).

\section{Safety and compliance}

Adverse events were reported by 45 patients ( $94 \%$ of included patients, $96 \%$ in the milnacipran group and $92 \%$ in the placebo group). These were mainly gastrointestinal disorders (28.4\% vs $29.6 \%)$, general disorders ( $14.7 \%$ vs $18.5 \%)$, and nervous system disorders (14.7\% vs $16.7 \%$ ) (Figure 6). No serious adverse event was reported. Compliance to treatment

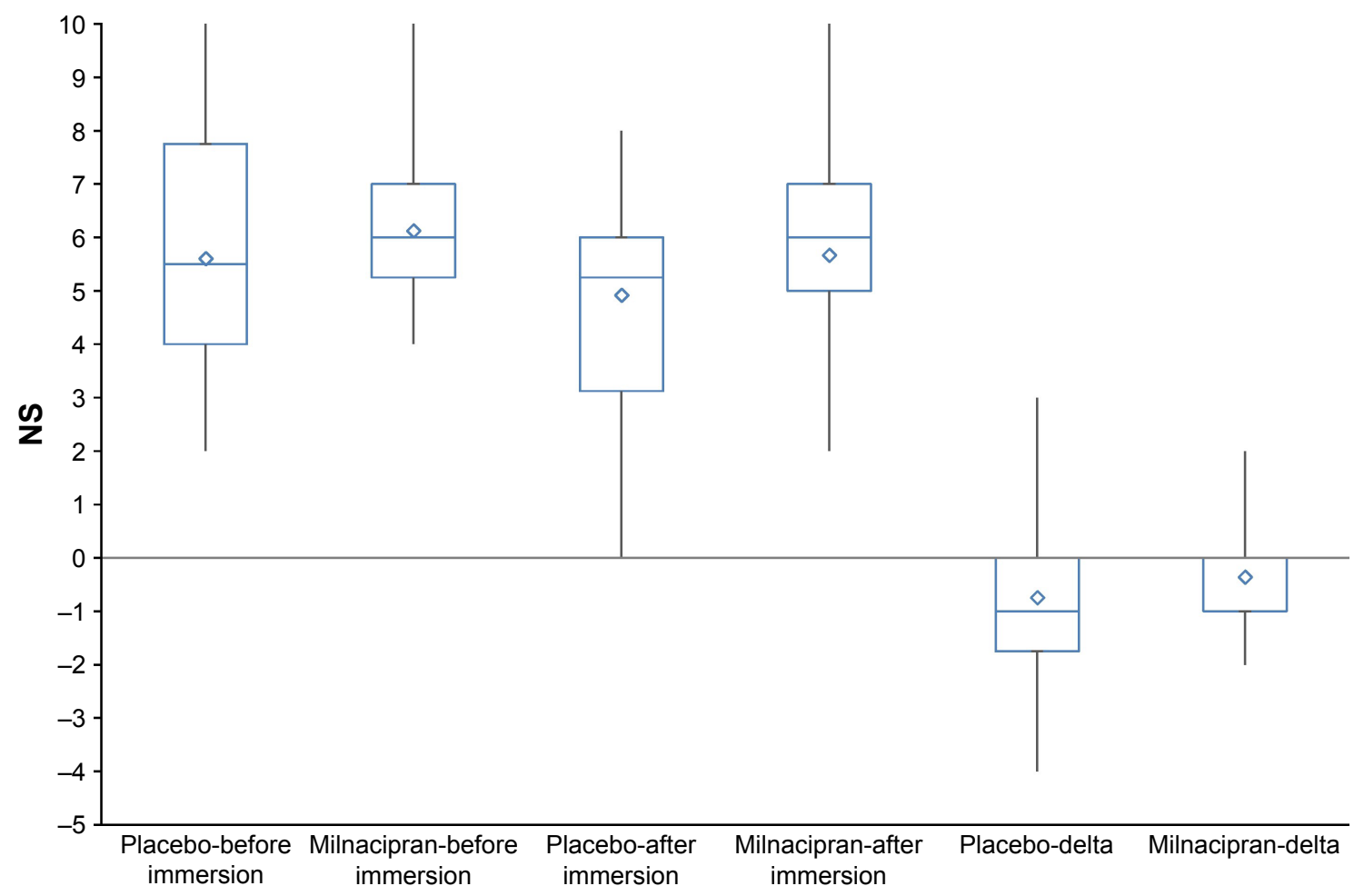

Figure 2 Pain evaluation (NRS) of 30-second conditioned pain modulation test at MI. Abbreviation: NRS, Numeric Pain Rating Scale. 


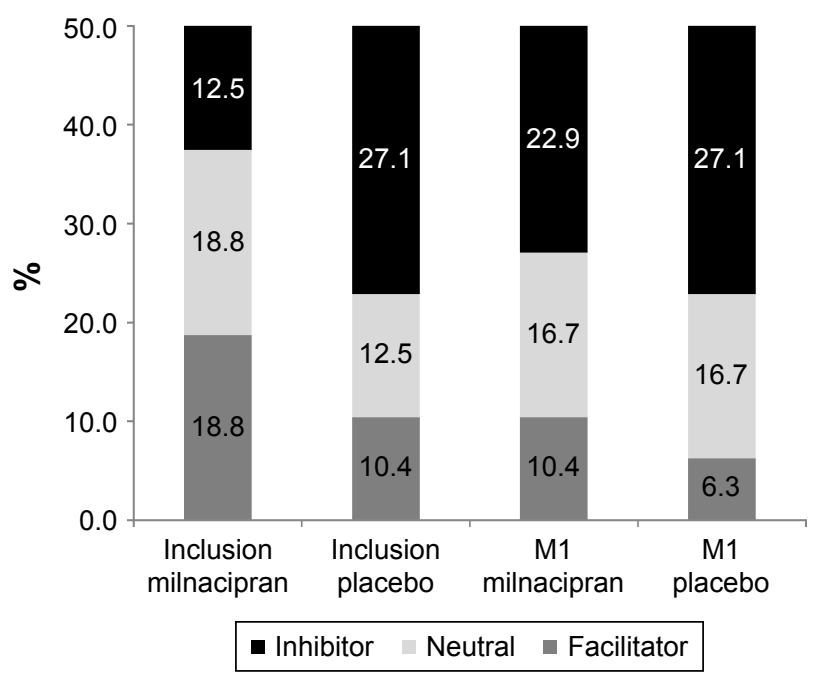

Figure 3 Percentage of patients according to the status of pain modulation profile (30-second conditioned pain modulation test) at inclusion and MI.

was $97.4 \%$ in the milnacipran group and $99.4 \%$ in the placebo group $(p=0.41)$.

\section{Discussion}

This randomized double-blind controlled trial conducted in patients suffering from FM did not show any significant reactivation of CPM with milnacipran compared with placebo after a strong painful CPM stimulation. It is, however, interesting to note a trend $(p=0.085)$ to develop an inhibitory

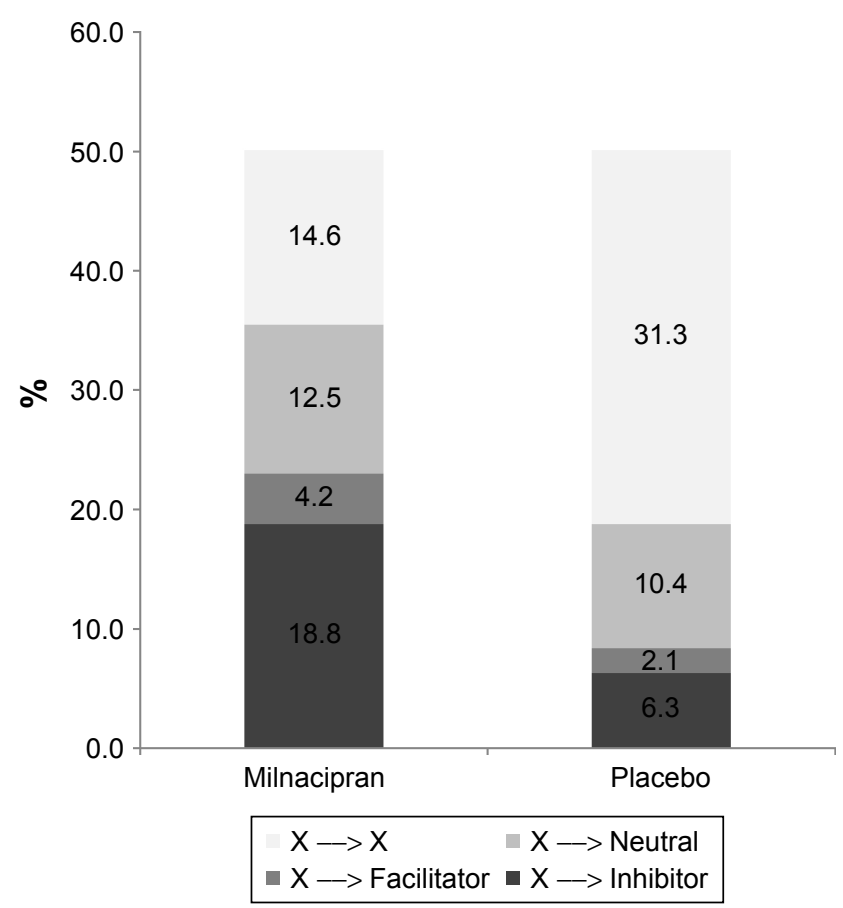

Figure 4 Pain modulation profile evolution (30-second conditioned pain modulation test) at MI. profile after 1-month treatment with milnacipran, with more patients becoming "inhibitory" in the milnacipran vs placebo group (18.8\% vs $6.3 \%$, Figure 4 ). While $40 \%$ patients had an inhibitory status at baseline, $29 \%$ were facilitators (Figure 3), a figure higher than the $21.2 \%$ described in the study by Potvin and Marchand, ${ }^{51}$ underlining the rather poor pain adaptation capability of FM patients. Similarly, no significant analgesic effect of milnacipran compared with placebo was observed on global pain, and pain diminished similarly in both groups, stressing the lack of efficacy of milnacipran and the strong placebo effect. This lack of analgesic effect is, however, contradictory to previous studies with milnacipran and duloxetine that showed ${ }^{40-43,56}$ a pain diminution of $30 \%$ in $40 \%$ patients, a value higher than that in our study (30\% diminution in $29 \%$ milnacipran group and $25 \%$ placebo group, $p=0.745$ ). The strong placebo effect observed in FM patients confirms, however, the literature on milnacipran ${ }^{60}$ and was also shown for duloxetine and gabapentin. Patients in the placebo group had a shorter FM history duration than those in the milnacipran group. Kosek et $\mathrm{al}^{61}$ reported that the shorter the duration of the history of FM, the stronger the placebo effect, suggesting a loss of placebo effect with time. Moreover, at baseline, while the dysfunction of CPM was obvious in both groups, a higher percentage of patients were classified as "inhibitor" in the placebo group (27.1\% vs $12.5 \%)$. Despite adequate randomization, patients in the placebo and milnacipran groups displayed these slight differences that may have contributed to diminish the effect of milnacipran according to covariance analysis.

Previous studies have shown that baseline pain modulation profile could be predictive of the efficacy of a treatment with duloxetine in patients with painful diabetic neuropathy. ${ }^{28}$ Our study did not show any correlation between CPM at baseline and CPM after treatment or global pain after treatment. This point is consistent with the heterogeneity of responses to milnacipran in FM patients, suggesting that subtypes of FM may be at play, as suggested by Yim et al. ${ }^{62}$ Knowledge of the pain modulation profile and characterization of FM subtype before treatment administration would allow to stratify patients to identify responders to milnacipran.

The study also investigated if cognitive function would be modified by milnacipran. Chronic pain and FM are known to have a deleterious influence on cognitivo-emotional domains, affecting emotion, concentration, and memory. ${ }^{44,56,63}$ Our FM sample was cognitively impaired regarding vigilance and memory when compared with non-FM healthy volunteers from a previous study (Corriger et al, unpublished data, 2017). 


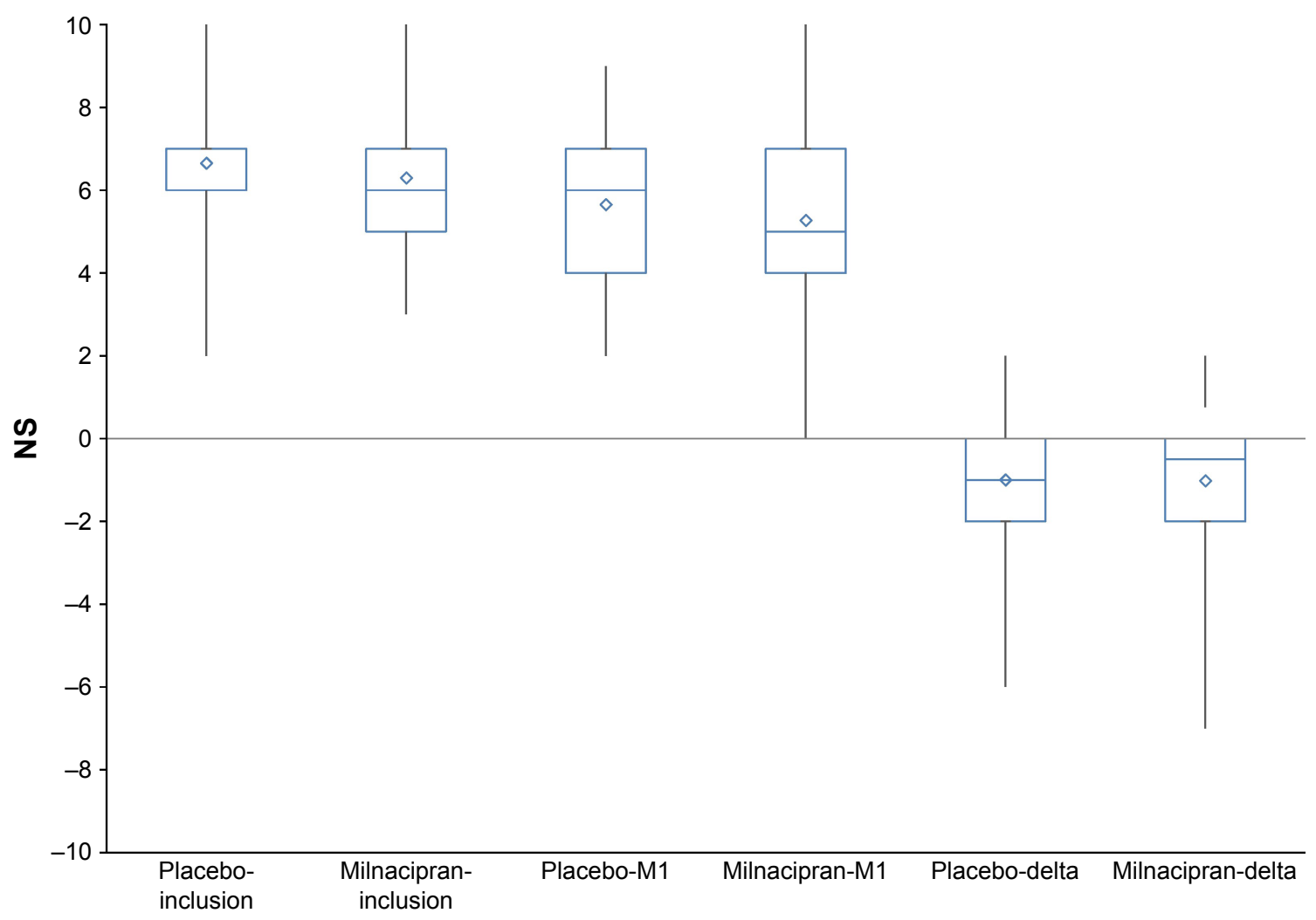

Figure 5 Global pain raw values (NRS) and differences at inclusion and MI.

Abbreviation: NRS, Numeric Pain Rating Scale.

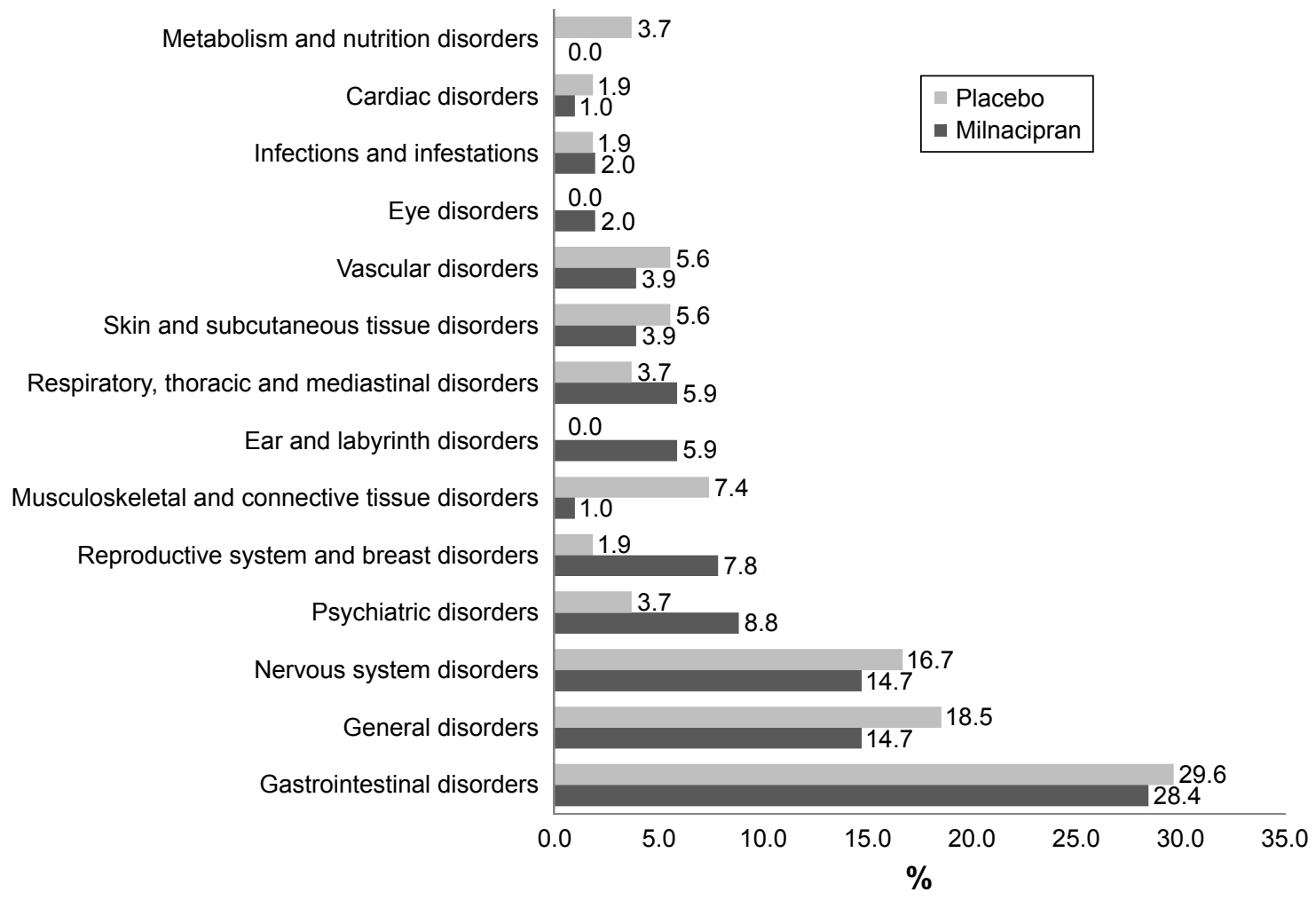

Figure 6 Reported adverse events. 
After 1-month treatment with milnacipran, no difference was, however, highlighted between both groups.

Finally, concerning tolerance, 96\% FM patients developed serotonergic AEs in the milnacipran group as reported in the literature. ${ }^{4,56,63}$ These AEs led to withdrawal from the protocol ( 5 patients in the milnacipran group, 1 in the placebo group), confirming data of a meta-analysis ${ }^{45}$ that reported that milnacipran $100 \mathrm{mg}$ daily was less well tolerated than duloxetine and pregabalin. However, FM patients with milnacipran reported AE described in the French drug label, ${ }^{64}$ confirming the good compliance of patients to their treatment.

\section{Limitations}

Among limitations of our study, the length of the treatment was limited to 1 month, as milnacipran efficacy has been reported within 1-3 weeks treatment. ${ }^{64}$ Another limitation concerns the fact that we included only women and no men in our experimental trial, in order to have a methodological homogeneity, although in real life the female:male ratio is $2: 1$.

\section{Conclusion}

A 1-month treatment with milnacipran did not show major reactivation of the functionality of $\mathrm{CPM}$, and placebo and nocebo effects were observed. As milnacipran is recommended for FM in several countries, ${ }^{65}$ it is important to stress the poor efficacy of milnacipran in the current trial, the occurrence of expected adverse events, and the large heterogeneity of FM patients concerning pain perception, pain modulation profile, and placebo effect. Stratification on CPM, inhibitory or facilitatory status, or FM history duration would allow to conclude on milnacipran pain alleviation in specific FM profiles. Larger clinical trials need to address these issues with multifactorial and multidimensional approaches to better subgroup FM patients to propose individualized therapeutic strategies.

\section{Acknowledgments}

We thank the Apicil foundation for their financial support, the patients for their participation, nurses S Boulliau, S Eschalier, and the team of the Clinical Research Center of Clermont-Ferrand, France, for their help in the realization of this study.

\section{Author contributions}

This clinical research was constructed by all authors. GP, FM, ND, PP, and BP conducted the study concept and design. GP, FM, ND, and PP preselected the patients suffering from fibromyalgia. GP and CD included the patients in the study. FG and NM realized the acquisition of data. GP, J-MC, CD, FM, BP, SS-A, and NM analyzed, interpreted, and wrote the first draft of the manuscript. All authors contributed toward data analysis, drafting and critically revising the paper and agree to be accountable for all aspects of the work.

\section{Disclosure}

The authors report no conflicts of interest in this work.

\section{References}

1. Perrot S, Choy E, Petersel D, Ginovker A, Kramer E. Survey of physician experiences and perceptions about the diagnosis and treatment of fibromyalgia. BMC Health Serv Res. 2012;12:356.

2. Branco JC, Bannwarth B, Failde I, et al. Prevalence of fibromyalgia: a survey in five European countries. Semin Arthritis Rheum. 2010;39(6): 448-453.

3. Corsalini M, Daniela DV, Biagio R, Gianluca S, Alessandra L, Francesco P. Evidence of signs and symptoms of craniomandibular disorders in fibromyalgia patients. Open Dent J. 2017;11(1):91-98.

4. Lawrence RC, Felson DT, Helmick CG, et al. Estimates of the prevalence of arthritis and other rheumatic conditions in the United States. Part II. Arthritis Rheum. 2008;58(1):26-35.

5. Thomas E, Missounga L, Blotman F. Fibromyalgie. EMC-Médecine. 2005;2(4):369-377.

6. Wolfe F, Clauw DJ, Fitzcharles M-A, et al. The American College of Rheumatology preliminary diagnostic criteria for fibromyalgia and measurement of symptom severity. Arthritis Care Res. 2010;62(5): 600-610.

7. Perrot S, Vicaut E, Servant D, Ravaud P. Prevalence of fibromyalgia in France: a multi-step study research combining national screening and clinical confirmation: the DEFI study (Determination of Epidemiology of FIbromyalgia). BMC Musculoskelet Disord. 2011;12:224.

8. Bidonde J, Boden C, Busch AJ, Goes SM, Kim S, Knight E. Dance for adults with fibromyalgia-what do we know about it? Protocol for a scoping review. JMIR Res Protoc. 2017;22;6(2):e25.

9. Mease PJ. Fibromyalgia, a missed comorbidity in spondyloarthritis: prevalence and impact on assessment and treatment. Curr Opin Rheumatol. 2017;29(4):304-310.

10. Bradley LA. Pathophysiology of fibromyalgia. Am J Med. 2009; 122(12 Suppl):S22-S30.

11. Jay GW, Barkin RL. Fibromyalgia. Dis - Mon DM. 2015;61(3): 66-111.

12. Cook DB, Lange G, Ciccone DS, Liu W-C, Steffener J, Natelson BH. Functional imaging of pain in patients with primary fibromyalgia. J Rheumatol. 2004;31(2):364-378.

13. Clauw DJ. Fibromyalgia: an overview. Am J Med. 2009;122(12 Suppl): S3-S13.

14. Gracely RH, Petzke F, Wolf JM, Clauw DJ. Functional magnetic resonance imaging evidence of augmented pain processing in fibromyalgia. Arthritis Rheum. 2002;46(5):1333-1343.

15. Foerster BR, Petrou M, Harris RE, et al. Cerebral blood flow alterations in pain-processing regions of patients with fibromyalgia using perfusion MR imaging. AJNR Am J Neuroradiol. 2011;32(10): 1873-1878.

16. Shokouhi M, Davis KD, Moulin DE, et al. Basal Ganglia perfusion in fibromyalgia is related to pain disability and disease impact: an arterial spin labeling study. Clin J Pain. 2016;32(6):495-505.

17. Mhalla A, Baudic S, Ciampi de Andrade D, et al. Long-term maintenance of the analgesic effects of transcranial magnetic stimulation in fibromyalgia. Pain. 2011;152(7):1478-1485. 
18. Kuchinad A, Schweinhardt P, Seminowicz DA, Wood PB, Chizh BA, Bushnell MC. Accelerated brain gray matter loss in fibromyalgia patients: premature aging of the brain? J Neurosci Off J Soc Neurosci. 2007; 27(15):4004-4007.

19. Robinson ME, Craggs JG, Price DD, Perlstein WM, Staud R. Gray matter volumes of pain-related brain areas are decreased in fibromyalgia syndrome. J Pain Off J Am Pain Soc. 2011;12(4):436-443.

20. Brooks L, Hadi J, Amber KT, Weiner M, La Riche CL, Ference T. Assessing the prevalence of autoimmune, endocrine, gynecologic, and psychiatric comorbidities in an ethnically diverse cohort of female fibromyalgia patients: does the time from hysterectomy provide a clue? J Pain Res. 2015;8:561-569.

21. Cerdá-Olmedo G, Mena-Durán AV, Monsalve V, Oltra E. Identification of a microRNA signature for the diagnosis of fibromyalgia. PLoS One. 2015;10(3):e0121903.

22. O'Mahony SM, Dinan TG, Cryan JF. The gut microbiota as a key regulator of visceral pain. Pain. 2017;158(Suppl 1):S19-S28.

23. Cagnie B, Coppieters I, Denecker S, Six J, Danneels L, Meeus M. Central sensitization in fibromyalgia? A systematic review on structural and functional brain MRI. Semin Arthritis Rheum. 2014;44(1):68-75.

24. Fallon N, Chiu Y, Nurmikko T, Stancak A. Functional connectivity with the default mode network is altered in fibromyalgia patients. PLoS One. 2016;11(7):e0159198.

25. Macian N, Pereira B, Shinjo C, Dubray C, Pickering G. Fibromyalgia, milnacipran and experimental pain modulation: study protocol for a double-blind randomized controlled trial. Trials. 2015;16:134.

26. Le Bars D, Villanueva L, Bouhassira D, Willer JC. Diffuse noxious inhibitory controls (DNIC) in animals and in man. Patol Fiziol Eksp Ter. 1992;4:55-65.

27. Moont R, Pud D, Sprecher E, Sharvit G, Yarnitsky D. "Pain inhibits pain" mechanisms: is pain modulation simply due to distraction? Pain. 2010;150(1):113-120.

28. Yarnitsky D, Granot M, Nahman-Averbuch H, Khamaisi M, Granovsky Y. Conditioned pain modulation predicts duloxetine efficacy in painful diabetic neuropathy. Pain. 2012;153(6):1193-1198.

29. Granovsky YG, Yarnitsky D. Personalized pain medicine: the clinical value of psychophysical assessment of pain modulation profile. Rambam Maimonides Med J. 2013;4(4):e0024.

30. Ichesco E, Puiu T, Hampson JP, et al. Altered fMRI resting-state connectivity in individuals with fibromyalgia on acute pain stimulation Eur J Pain. 2016;20(7):1079-1089.

31. Flodin P, Martinsen S, Löfgren M, Bileviciute-Ljungar I, Kosek E, Fransson P. Fibromyalgia is associated with decreased connectivity between pain- and sensorimotor brain areas. Brain Connect. 2014;4(8) 587-594.

32. Emad Y, Ragab Y, Zeinhom F, El-Khouly G, Abou-Zeid A, Rasker JJ. Hippocampus dysfunction may explain symptoms of fibromyalgia syndrome. A study with single-voxel magnetic resonance spectroscopy. J Rheumatol. 2008;35(7):1371-1377.

33. Fayed N, Garcia-Campayo J, Magallón R, et al. Localized 1H-NMR spectroscopy in patients with fibromyalgia: a controlled study of changes in cerebral glutamate/glutamine, inositol, choline, and N-acetylaspartate. Arthritis Res Ther. 2010;12(4):R134.

34. Fayed N, Andres E, Rojas G, et al. Brain dysfunction in fibromyalgia and somatization disorder using proton magnetic resonance spectroscopy: a controlled study. Acta Psychiatr Scand. 2012;126(2): $115-125$.

35. Feraco P, Bacci A, Pedrabissi F, et al. Metabolic abnormalities in painprocessing regions of patients with fibromyalgia: a 3T MR spectroscopy study. AJNR Am J Neuroradiol. 2011;32(9):1585-1590.

36. Foerster BR, Nascimento TD, DeBoer M, et al. Excitatory and inhibitory brain metabolites as targets of motor cortex transcranial direct current stimulation therapy and predictors of its efficacy in fibromyalgia. Arthritis Rheumatol. 2015;67(2):576-581.

37. Harris RE, Sundgren PC, Craig AD, et al. Elevated insular glutamate in fibromyalgia is associated with experimental pain. Arthritis Rheum. 2009;60(10):3146-3152.
38. Natelson BH, Vu D, Mao X, et al. Effect of Milnacipran treatment on ventricular lactate in fibromyalgia: a randomized, double-blind, placebo-controlled trial. J Pain Off J Am Pain Soc. 2015;16(11): 1211-1219.

39. Petrou M, Harris RE, Foerster BR, et al. Proton MR spectroscopy in the evaluation of cerebral metabolism in patients with fibromyalgia: comparison with healthy controls and correlation with symptom severity. AJNR Am J Neuroradiol. 2008;29(5):913-918.

40. Lee YH, Song GG. Comparative efficacy and tolerability of duloxetine, pregabalin, and milnacipran for the treatment of fibromyalgia: a Bayesian network meta-analysis of randomized controlled trials. Rheumatol Int. 2016;36(5):663-672.

41. Leventhal L, Smith V, Hornby G, Andree TH, Brandt MR, Rogers KE. Differential and synergistic effects of selective norepinephrine and serotonin reuptake inhibitors in rodent models of pain. J Pharmacol Exp Ther. 2007;320(3):1178.

42. Suzuki R, Rygh LJ, Dickenson AH. Bad news from the brain: descending 5-HT pathways that control spinal pain processing. Trends Pharmacol Sci. 2004;25(12):613-617.

43. Blakely RD, Bauman AL. Biogenic amine transporters: regulation in flux. Curr Opin Neurobiol. 2000;10(3):328-336.

44. Millan MJ. Serotonin (5-HT) and pain: a reappraisal of its role in the light of receptor multiplicity. Semin Neurosci. 1995;7(6):409-419.

45. Millan MJ. Descending control of pain. Prog Neurobiol. 2002; 66(6):355-474.

46. Cording M, Derry S, Phillips T, Moore RA, Wiffen PJ. Milnacipran for pain in fibromyalgia in adults. Cochrane Database Syst Rev. 2015;10: CD008244.

47. Carville SF, Arendt-Nielsen S, Bliddal H, et al. EULAR evidence-based recommendations for the management of fibromyalgia syndrome. Ann Rheum Dis. 2008;67(4):536.

48. Bradley LA, Wohlreich MM, Wang F, et al. Pain response profile of patients with fibromyalgia treated with duloxetine. Clin J Pain. 2010;26(6):498-504.

49. Russell IJ, Mease PJ, Smith TR, et al. Efficacy and safety of duloxetine for treatment of fibromyalgia in patients with or without major depressive disorder: results from a 6-month, randomized, double-blind, placebo-controlled, fixed-dose trial. Pain. 2008;136(3): 432-444.

50. Branco JC, Cherin P, Montagne A, Bouroubi A. Longterm therapeutic response to Milnacipran treatment for fibromyalgia. A European 1-year extension study following a 3-month study. J Rheumatol. 2011; 1403-1412.

51. Potvin S, Marchand S. Pain facilitation and pain inhibition during conditioned pain modulation in fibromyalgia and in healthy controls. Pain. 2016;157(8):1704-1710.

52. Wolfe F, Clauw DJ, Fitzcharles MA, et al. The American College of Rheumatology preliminary diagnostic criteria for fibromyalgia and measurement of symptom severity. Arthritis Care Res (Hoboken). 2010;62(5):600-610.

53. Pickering G, Pereira B, Dufour E, Soule S, Dubray C. Impaired modulation of pain in patients with postherpetic neuralgia. Pain Res Manag. 2014;19(1):e19-e23.

54. Granovsky Y, Matre D, Sokolik A, Lorenz J, Casey KL. Thermoreceptive innervation of human glabrous and hairy skin: a contact heat evoked potential analysis. Pain. 2005;115(3):238-247.

55. Julien N, Goffaux P, Arsenault P, Marchand S. Widespread pain in fibromyalgia is related to a deficit of endogenous pain inhibition. Pain. 2005;114(1-2):295-302.

56. Lee YC, Massarotti E, Edwards RR, et al. The effect of Milnacipran on pain in rheumatoid arthritis patients with widespread pain: a randomized blinded crossover trial. J Rheumatol. 2016;43(1):38-45.

57. Yarnitsky D, Granot M, Granosky Y. Pain modulation profile and pain therapy: between pro- and antinociception. Pain. 2014;155(4):663-665

58. Pickering G, Pereira B, Clère F, et al. Cognitive function in older patients with postherpetic neuralgia. Pain Pract Off J World Inst Pain. 2014;14(1):E1-E7. 
59. Vickers AJ, Altman DG. Statistics notes: analysing controlled trials with baseline and follow up measurements. BMJ. 2001;323(7321): 1123-1124.

60. Arnold LM, Gendreau RM, Palmer RH, Gendreau JF, Wang Y. Efficacy and safety of milnacipran $100 \mathrm{mg} /$ day in patients with fibromyalgia: results of a randomized, double-blind, placebo-controlled trial. Arthritis Rheum. 2010;62(9):2745-2756.

61. Kosek E, Rosen A, Carville S, et al. Lower placebo responses after longterm exposure to fibromyalgia pain. J Pain. 2017;18(7):835-843.

62. Yim Y-R, Lee K-E, Park D-J, et al. Identifying fibromyalgia subgroups using cluster analysis: relationships with clinical variables. Eur J Pain. 2017;21(2):374-384.
63. Arnold LM, Bateman L, Palmer RH, Lin Y. Preliminary experience using milnacipran in patients with juvenile fibromyalgia: lessons from a clinical trial program. Pediatr Rheumatol Online J. 2015;13:27.

64. Milnacipran (IXEL ${ }^{\circledR}$ ) Résumé caractéristiques produit. ANSM. 2017; Available from: http://agence-prd.ansm.sante.fr/php/ecodex/rcp/ R0223835.htm. Accessed September 25, 2017.

65. Liu Y, Qian C, Yang M. Treatment patterns associated with ACRrecommended medications in the management of fibromyalgia in the United States. J Manag Care Spec Pharm. 2016;22(3):263-271. 


\section{Supplementary materials}

Table SI Demographic and baseline characteristics of patient at inclusion

\begin{tabular}{|c|c|c|c|}
\hline Parameter/test & $\begin{array}{l}\text { Milnacipran, } \\
\mathrm{n}=24\end{array}$ & $\begin{array}{l}\text { Placebo, } \\
n=24\end{array}$ & p-value \\
\hline FM duration (months), mean \pm SD & $91.3 \pm 86.0$ & $52.4 \pm 33.3$ & 0.048 \\
\hline Age (years), mean $\pm S D$ & $48.0 \pm 11.0$ & $44.3 \pm 9.3$ & 0.210 \\
\hline Weight (kg), mean $\pm S D$ & $67.6 \pm 14.1$ & $72.3 \pm 16.1$ & 0.295 \\
\hline Treatment, $\mathrm{n}(\%)$ & $23(95.8)$ & $23(95.8)$ & 1.000 \\
\hline Global pain, numerical scale, NRS, mean $\pm S D$ & $6.3 \pm 2.0$ & $6.6 \pm 1.6$ & 0.501 \\
\hline Pain $\left(\mathrm{CPM}_{10}\right)$, before immersion, NRS, mean $\pm \mathrm{SD}$ & $6.0 \pm 1.22$ & $5.96 \pm 1.05$ & 0.900 \\
\hline Pain $\left(\mathrm{CPM}_{10}\right)$, after immersion, NRS, mean $\pm \mathrm{SD}$ & $5.9 \pm 1.9$ & $5.9 \pm 1.2$ & 0.927 \\
\hline $\mathrm{CPM}_{10}$, mean $\pm \mathrm{SD}$ & $-0.06 \pm 1.90$ & $-0.06 \pm 1.34$ & 1.000 \\
\hline Pain $\left(\mathrm{CPM}_{30}\right)$, before immersion $30 \mathrm{~s}, \mathrm{NRS}$, mean $\pm \mathrm{SD}$ & $5.9 \pm 1.65$ & $6.4 \pm 1.5$ & 0.223 \\
\hline Pain $\left(\mathrm{CPM}_{30}\right)$, after immersion $30 \mathrm{~s}$, NRS, mean $\pm \mathrm{SD}$ & $5.8 \pm 2.3$ & $5.4 \pm 2.5$ & 0.506 \\
\hline $\mathrm{CPM}_{30}$, mean $\pm \mathrm{SD}$ & $-0.02 \pm 1.52$ & $-1.04 \pm 2.15$ & 0.065 \\
\hline \multicolumn{4}{|l|}{ Pain modulation profile } \\
\hline Inhibitors, $\mathrm{CPM}_{10}, \mathrm{n}(\%)$ & $7(14.6)$ & $10(20.8)$ & \\
\hline Facilitator, $\mathrm{CPM}_{10}, \mathrm{n}(\%)$ & $8(16.7)$ & $8(16.7)$ & 0.589 \\
\hline Neutral, $\mathrm{CPM}_{10}, \mathrm{n}(\%)$ & $9(18.8)$ & $6(12.5)$ & \\
\hline Inhibitors, $\mathrm{CPM}_{30}, \mathrm{n}(\%)$ & $6(12.5)$ & $13(27.1)$ & 0.131 \\
\hline Facilitator, $\mathrm{CPM}_{30}, \mathrm{n}(\%)$ & $9(18.8)$ & $5(10.4)$ & \\
\hline Neutral, $\mathrm{CPM}_{30}, \mathrm{n}(\%)$ & $9(18.8)$ & $6(12.5)$ & \\
\hline Thermal heat pain sensitivity, ${ }^{\circ} \mathrm{C}$, mean $\pm \mathrm{SD}$ & $35.0 \pm 1.1$ & $34.5 \pm 0.6$ & 0.077 \\
\hline Thermal cold pain sensitivity, ${ }^{\circ} \mathrm{C}$, mean $\pm \mathrm{SD}$ & $29.9 \pm 0.9$ & $29.9 \pm 1.3$ & 0.942 \\
\hline Thermal heat pain threshold, ${ }^{\circ} \mathrm{C}$, mean $\pm \mathrm{SD}$ & $39.7 \pm 2.9$ & $40.4 \pm 3.7$ & 0.508 \\
\hline Thermal cold pain threshold, ${ }^{\circ} \mathrm{C}$, mean $\pm \mathrm{SD}$ & $24.5 \pm 5.0$ & $24.2 \pm 5.5$ & 0.846 \\
\hline Thermal heat pain threshold $(\mathrm{T} 6 / 10),{ }^{\circ} \mathrm{C}$, mean $\pm \mathrm{SD}$ & $41.9 \pm 4.0$ & $41.4 \pm 4.2$ & 0.765 \\
\hline Mechanical allodynia, NRS, mean \pm SD & $0.8 \pm 1.2$ & $0.8 \pm 2.0$ & 0.912 \\
\hline Mechanical sensitivity, von Frey ${ }^{\circledR}$, mean \pm SD & $3.5 \pm 0.4$ & $3.5 \pm 0.3$ & 0.507 \\
\hline $\begin{array}{l}\text { Mechanical temporal summation, von Frey, NRS, } \\
\text { mean } \pm \text { SD }\end{array}$ & $2.0 \pm 1.8$ & $2.3 \pm 1.6$ & 0.614 \\
\hline
\end{tabular}

Abbreviations: CPM, conditioned pain modulation; $\mathrm{CPM}_{10}$, CPM for 10 seconds stimulation; $\mathrm{CPM}_{30}$, CPM for 30 seconds stimulation; FM, fibromyalgia; NRS, Numeric Pain Rating Scale.

Table S2 Cantab $^{\circledR}$ baseline characteristics of fibromyalgia patient at inclusion

\begin{tabular}{|c|c|c|c|c|c|}
\hline Test, mean \pm SD & $\mathbf{n}$ & Milnacipran & $\mathbf{n}$ & Placebo & $p$-value \\
\hline SOC MITT & 23 & $7,047.6 \pm 6,200.6$ & 24 & $9,836.7 \pm 7,030.8$ & 0.160 \\
\hline SOC MSTT & & $3,295.9 \pm 4,352.8$ & & $2,997.1 \pm 4,369.3$ & 0.817 \\
\hline SOC PSMV & & $7.1 \pm 2.5$ & & $7.5 \pm 2.3$ & 0.564 \\
\hline RTI FCRT & 24 & $424.6 \pm 101.2$ & 24 & $442.1 \pm 135.3$ & 0.617 \\
\hline RTI FCMT & & $518.9 \pm 120.4$ & & $507.9 \pm 136.4$ & 0.768 \\
\hline CGT QDM & 23 & $0.85 \pm 0.14$ & 24 & $0.82 \pm 0.17$ & 0.465 \\
\hline CGT DT & & $2,734.3 \pm 893.3$ & & $2,952.2 \pm 1,003.2$ & 0.435 \\
\hline CGT RT & & $0.47 \pm 0.18$ & & $0.45 \pm 0.14$ & 0.715 \\
\hline CGT RA & & $0.58 \pm 0.83$ & & $0.76 \pm 0.79$ & 0.427 \\
\hline CGT DA & 23 & $0.42 \pm 0.25$ & 24 & $0.34 \pm 0.21$ & 0.258 \\
\hline CGT OPB & & $0.44 \pm 0.18$ & & $0.43 \pm 0.14$ & 0.768 \\
\hline GNT TC & 23 & $21.3 \pm 4.6$ & 24 & $21.4 \pm 3.3$ & 0.972 \\
\hline GNT TE & & $8.7 \pm 4.6$ & & $8.6 \pm 3.3$ & 0.972 \\
\hline GNT PC & & $7 I .1 \pm 15.2$ & & $71.2 \pm 11.0$ & 0.972 \\
\hline
\end{tabular}

Abbreviations: CGT, Cambridge Gambling Task; DA, delay aversion; DT, deliberation time; FCMT, five-choice movement time; FCRT, five-choice reaction time; GNT, Graded Naming Test; MITT, mean initial thinking time; MSTT, mean subsequent thinking time; OPB, overall proportion bet; PC, percent correct; PSMV, problems solved in minimum moves; QDM, quality of decision making; RA, risk adjustment; RT, risk taking; RTI, reaction time; SOC, Stockings of Cambridge; TC, total correct; TE, total errors. 
Table S3 Comparison between groups at MI

\begin{tabular}{|c|c|c|c|c|c|}
\hline Test & $\mathbf{n}$ & Milnacipran & $\mathbf{n}$ & Placebo & $p$-value \\
\hline Thermal heat pain sensitivity, ${ }^{\circ} \mathrm{C}$, mean $\pm \mathrm{SD}$ & 24 & $34.6 \pm 0.7$ & 24 & $34.7 \pm 1.0$ & 0.900 \\
\hline Thermal cold pain sensitivity, ${ }^{\circ} \mathrm{C}$ mean \pm SD & & $29.6 \pm 1.3$ & & $30.0 \pm 1.0$ & 0.298 \\
\hline Thermal heat pain threshold, ${ }^{\circ} \mathrm{C}$, mean $\pm \mathrm{SD}$ & & $39.7 \pm 3.2$ & & $40.5 \pm 3.7$ & 0.438 \\
\hline Thermal cold pain threshold, ${ }^{\circ} \mathrm{C}$, mean $\pm \mathrm{SD}$ & & $23.5 \pm 6.1$ & & $22.5 \pm 6.4$ & 0.598 \\
\hline Mechanical sensitivity, mean $\pm S D$ & & $3.48 \pm 0.46$ & & $3.48 \pm 0.39$ & 0.680 \\
\hline Mechanical temporal summation, NRS, mean \pm SD & & $2.29 \pm 1.4$ & & $2.04 \pm 1.63$ & 0.571 \\
\hline Thermal heat pain threshold $(\mathrm{T} 6 / \mathrm{I} 0),{ }^{\circ} \mathrm{C}$, mean $\pm \mathrm{SD}$ & & $42.3 \pm 3.9$ & & $41.8 \pm 3.9$ & 0.599 \\
\hline Mechanical allodynia, NRS, mean \pm SD & & $1.63 \pm 2.18$ & & $1.21 \pm 2.34$ & 0.509 \\
\hline SOC MITT & 23 & $6,155.2 \pm 6,657.1$ & 24 & $5,758.1 \pm 4,508.9$ & 0.404 \\
\hline SOC MSTT & & $|, 608.6 \pm I, 8| 8 . \mid$ & & $\mathrm{I}, 395.7 \pm 1,340.1$ & 0.726 \\
\hline SOC PSMV & & $7.9 \pm 2.0$ & & $8.4 \pm 1.8$ & 0.422 \\
\hline RTI FCRT & 23 & $430.4 \pm 303.6$ & 24 & $416.5 \pm 98.9$ & 0.946 \\
\hline RTI FCMT & & $517.9 \pm 105.8$ & & $495.6 \pm 127.0$ & 0.527 \\
\hline CGT QDM & 22 & $0.89 \pm 0.13$ & 24 & $0.84 \pm 0.16$ & 0.298 \\
\hline CGT DT & 22 & $2,308.1 \pm 988.2$ & 24 & $2,378.4 \pm 929.7$ & 0.605 \\
\hline CGT RT & & $0.50 \pm 0.17$ & & $0.48 \pm 0.15$ & 0.529 \\
\hline CGT RA & & $0.60 \pm 0.88$ & & $0.90 \pm 0.95$ & 0.249 \\
\hline CGT DA & & $0.34 \pm 0.22$ & & $0.33 \pm 0.22$ & 0.567 \\
\hline CGT OPB & & $0.47 \pm 0.16$ & & $0.45 \pm 0.15$ & 0.560 \\
\hline GNT TC & 23 & $23.1 \pm 5.0$ & 24 & $23.2 \pm 3.7$ & 0.960 \\
\hline GNT TE & & $6.9 \pm 5.0$ & & $6.8 \pm 3.7$ & 0.960 \\
\hline GNT PC & & $77.1 \pm 16.5$ & & $77.2 \pm 12.4$ & 0.959 \\
\hline
\end{tabular}

Abbreviations: CGT, Cambridge Gambling Task; DA, delay aversion; DT, deliberation time; FCMT, five-choice movement time; FCRT, five-choice reaction time; GNT, Graded Naming Test; MITT, mean initial thinking time; MSTT, mean subsequent thinking time; NRS, Numeric Pain Rating Scale; OPB, overall proportion bet; PC, percent correct; PSMV, problems solved in minimum moves; QDM, quality of decision making; RA, risk adjustment; RT, risk taking; RTI, reaction time; SOC, Stockings of Cambridge; TC, total correct; TE, total errors.

\section{Publish your work in this journal}

Drug Design, Development and Therapy is an international, peerreviewed open-access journal that spans the spectrum of drug design and development through to clinical applications. Clinical outcomes, patient safety, and programs for the development and effective, safe, and sustained use of medicines are the features of the journal, which has also been accepted for indexing on PubMed Central. The manuscript management system is completely online and includes a very quick and fair peer-review system, which is all easy to use. Visit http://www.dovepress.com/testimonials.php to read real quotes from published authors. 